\title{
A Deep Learning Method for Prediction of Cardiovascular Disease Using Convolutional Neural Network
}

\author{
Tulasi Krishna Sajja*, Hemantha Kumar Kalluri
}

Vignan's Foundation for Science, Technology and Research deemed to be University, Vadlamudi, Guntur 522213, AP, India

Corresponding Author Email: stk_cse@vignan.ac.in

https://doi.org/10.18280/ria.340510

Received: 22 June 2020

Accepted: 3 October 2020

\section{Keywords:}

cardiovascular disease, heart attack, convolutional neural network, SVM, KNN, logistic regression, nä̈ve Bayes, deep learning

\begin{abstract}
Heart disease is a very deadly disease. Worldwide, the majority of people are suffering from this problem. Many Machine Learning (ML) approaches are not sufficient to forecast the disease caused by the virus. Therefore, there is a need for one system that predicts disease efficiently. The Deep Learning approach predicts the disease caused by the blocked heart. This paper proposes a Convolutional Neural Network (CNN) to predict the disease at an early stage. This paper focuses on a comparison between the traditional approaches such as Logistic Regression, K-Nearest Neighbors (KNN), Naïve Bayes (NB), Support Vector Machine (SVM), Neural Networks (NN), and the proposed prediction model of CNN. The UCI machine learning repository dataset for experimentation and Cardiovascular Disease (CVD) predictions with $94 \%$ accuracy.
\end{abstract}

\section{INTRODUCTION}

As per the latest survey by the WHO, $17.9 \mathrm{M}$ people pass away every year. There is no surprise that by the year 2030 it will increase to 75 million. American Heart Association considers approximately every 40 seconds, and an American will have a heart attack. Many cardiovascular diseases (CVD) exist to kill humans by using detectable hazards such as tobacco usages, unwanted eating habits, physical dormancy, and deadly usage of liquor in a range of contexts. Human beings who are having CVD are at high risk of cardiac. The disease requires early detection and guidance on the use of short medications, as set out below. Overall, CVD comes to an end with fatty stores' production within the ducts and blood groups' output. It can also be linked to an injury to tissues, such as the head, eyes, heart, and kidneys. CVD is a major leading cause of death and injury in the United Kingdom [1] but can be stopped daily to a wide degree by maintaining a good lifestyle. Cardiac cases and strokes are usually caused by powerful events and are mostly caused by a clot that prevents blood flow to the mind or heart. The most commonly known aim behind this is the creation of the most inward-looking greasy shops.

This issue had created a lot of seriousness between researchers; one of the critical tasks in this is to predict the disease present in the human body. Even doctors are also not efficient in predicting the disease [2]. However, they need a support system to predict the disease. Some of the algorithms are supported but need to improvise the system's performance beyond the existing system. Therefore, to help medicos, there is enormous research scope in predicting CVD disease in humans as support of medicos.

The Machine Learning algorithms are That type of support system proposed in this paper with deep learning technique. One of the deep learning algorithms, i.e., convolutional network (CNN) diagnose disease better than the existing methods. This CNN based model deals with a high volume of data. The advantage of $\mathrm{CNN}$ is everything is to be done by the network, such as preprocessing, feature extraction, prediction. The system accepts raw data.

The rest of the manuscript structured as follows: Section-2 describes the existing literature work. Section-3 explains the background of the machine learning algorithms and their functionalities. Section-4 gives an overview of the proposed methodology and also discussed the experimental results. Finally, conclusions in Section-5.

\section{RELATED WORK}

Beunza et al. [3] studied the clinical machine learning approaches regarding their validity and accuracy, logistic regression, Random Forest, decision tree, SVM, and NN. Zhao et al. [4] carefully studied the cardiovascular break-down rate with pulse transition by time analysis, ML, and CNN models used for examination. The support vector machine outperformed each of the classification algorithms in the assessment of cardiovascular recognition. Chen et al. [5] expected to calculate one year of cardiovascular events in patients with severe DCM using ML models. The ML algorithm's contribution was 32 highlights from clinical information, and Information Gain (IG) selected significant highlights that were exceptionally relevant to cardiovascular events. The study [6] explored the cardiovascular infections in humans on medications. Two ML techniques have been used and taken into account by Base of Fisiologiab Clinica; the other one was an American dataset supported regional diabetes and the Stomach Associated Association and the Vault of kidney diseases.

Awan et al. [7] expected the usage of the Artificial Neural Network for heart infections. The goal is to use machine learning and pattern matching strategies to fix heart disease. Gjoreski et al. [8] predicted the Continuous Cardiovascular Disruption Detection of Heart Rhythm utilizing ML 
classifiers' collection. These techniques used to predict include sampling, segmentation, selection of functions. Cardiovascular Disease Risk Prediction Approach using computerized machine learning has been given in the study [9]. An ML built model derived by the usage of auto-prognosis and an algorithmic method that, as a consequence, selects and implements the researchers used the ML simulation pipelines. Another ML method for the accurate determination of coronary artery disease was introduced by Abdar et al. [10]. The progression method, named as N2 Genetic Optimizer Agent, was introduced in this methodology. Such tests are violent and essentially the same as the most robust findings in the sector.

Tang et al. [11] provided the Continuous Arrhythmia heartbeat recognition algorithm. This method used in this treatment is the parallel delta Modulations and Rotated Linear SVM. Photonic crystal enhanced fluorescence visualization Cardiovascular disorder immunoassay biomarker test with machine learning investigation was conducted by Squire et al. [12]. PCA [13], PLSR Regression algorithms, and advanced ML Algorithm strategies are used in this study. Coronary artery disease, which is machine-based learning, was discussed by Alizadehsani et al. [14]; datasets tested, analyzed the weights, implementation measurements, and ML are the essential approaches that have been shattered down in this method. Research [15] used machine learning classifiers. The anticipation of hepatitis investigation, random forest classifier outflanked each of the classification models examined. Sajja et al. [16] proposed a medical assistive support system to classify the malignant and benign from lung CT scan images.

\section{BACKGROUND METHODS}

\subsection{Logistic regression}

It is one of the supervised learning and is used to estimate the target object value's possibility. It is a tool to calculate the statistical values and make results on binary output. In the linear method, which is calculated by the equation:

$$
y=b_{0}(x)+\mathrm{b}_{1}
$$

whereas logistic regression [17] used in Eq. (1). In this, the constant b1 bias and the slope b0 tell the curve's steepness.

$$
\text { Prob }=\frac{1}{1+e^{-(y)}}
$$

Here, $\mathrm{y}$ is the linear model's output trained with logistic regression produce value between zero and one.

\subsection{Naive Bayes}

In the Naïve Bayes network [18], all features are independent. When there is a change in one feature, it does not affect another. This is suitable for large datasets. The assumption from Conditional independence is that an attribute value is independent of the values, which are from other attribute values in a class. Bayes' Theorem [2] is based on probability theory:

$$
\operatorname{prob}(\mathrm{x} \mid \mathrm{y})=\frac{\operatorname{prob}(\mathrm{x}) \operatorname{prob}(\mathrm{y} \mid \mathrm{x})}{\operatorname{prob}(\mathrm{y})}
$$

where,

prob (x|y) - $\mathrm{x}$ happens given that $\mathrm{y}$ happens, $\operatorname{prob}(y \mid x)-y$ happens given that $\mathrm{x}$ happens, $\operatorname{prob}(\mathrm{x})-\mathrm{x}$ is on its own, $\operatorname{prob}(\mathrm{y})-\mathrm{y}$ is on its own.

\subsection{Support Vector Machine (SVM)}

SVM [19] is used both for regression and classification tasks. The SVM model represents the data in the space described so that the examples in various categories are divided by a distance as large as possible. That divides sensitive information with the maximum separable space between them and is calculated so that many of the points belong to one group fall on the plane's one side. This paper used two types of SVM kernels they are

\subsubsection{Linear kernel}

The dot product is performed among two observations. The equation of the linear kernel is in Eq. (4).

$$
\operatorname{kernel}\left(\mathrm{s}, \mathrm{si}_{\mathrm{i}}\right)=\operatorname{sum}\left(\mathrm{s}^{*} \mathrm{~s}_{\mathrm{i}}\right)
$$

From Eq. (4), observe that the product between two vectors, let say $\mathrm{s}$ and $\mathrm{s}_{\mathrm{i}}$, is the sum of the input values pair's multiplication.

\subsubsection{Radial Basis Function (RBF)}

$\mathrm{RBF}$ is mainly used in SVM classification, which maps input space in new dimensional space. Eq. (5) explains as

$$
\operatorname{Kernel}\left(\mathrm{s}, \mathrm{s}_{\mathrm{i}}\right)=\exp \left(-\operatorname{gamma} * \operatorname{sum}\left(\mathrm{s}^{-} \mathrm{s}_{\mathrm{i}}^{2}\right)\right)
$$

Generally, a gamma range between 0 to 1 . However, the default value for gamma is 0.1 .

\subsection{KNN}

K-Nearest Neighbor [20] is an anti-parametric method, which is used for regression and classification. It is essentially a grouping method, consider the distance between a point and the coordinates $(\mathrm{x}, \mathrm{y})$ and its neighbors. The distance between the Euclidean is in Eq. (6) and its neighbors are determined from the point and eventually located in the region nearest to its neighboring points.

$$
\text { Euclidean distance } D(x, y)=\sqrt{\sum_{i=1}^{n}\left(x_{i}-y_{i}\right)^{2}}
$$

Here, $\mathrm{x}, \mathrm{y}$ are two points in Euclidean ' $\mathrm{n}$ ' space, $x_{i}, y_{i}$ are two vectors starting from the initial point, ' $n$ ' means $n$ dimensional space.

\section{PROPOSED METHODOLOGY \& EXPERIMENTAL RESULTS}

\subsection{Data set}

For the experimentation, we considered Cleveland Heart Disease Dataset [21] from the UCI ML repository. This database contains 14 features; those are a person's age, gender, 
chest pain, treetops, chol, fbs, restecg, thalch, exang, oldpeak, slope, ca, thal and target. These 14 features are measured on 303 instances of heart disease patients. The particulars of the dataset were placed in Table 1.

\subsection{Methodology}

\subsubsection{Preprocessing}

At the principal level stage, the dataset is first cleaned and processed using preprocessing techniques using panda's package. The counterplot of sex and target attributes group is shown in Figure 1.

After that, using the data visualization procedure, the data frame attributes are shown in Figure 2, as histograms.

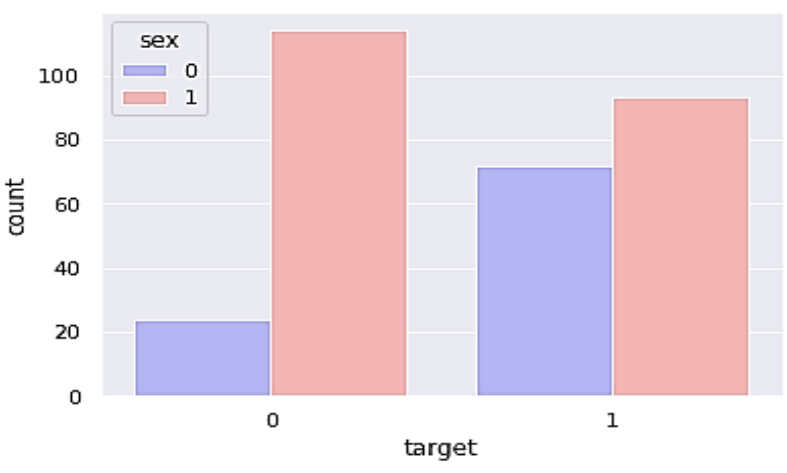

Figure 1. Counter plot grouped by sex vs. target

Table 1. Cleveland dataset details

\begin{tabular}{cccc}
\hline Attribute Name & Description & Value Type & Values Range \\
\hline Age & in years & Numerical & \\
sex & Gender info & Nominal & $1-\mathrm{m}, 0-\mathrm{f}$ \\
Chest pain & Type of cp & Nominal & $1,2,3,4$ \\
trestbps & resting blood pressure & Numerical & \\
chol & cholestoral $(\mathrm{mg} / \mathrm{dl})$ & Numerical & \\
fbs & fasting blood sugar $>(120 \mathrm{mg} / \mathrm{dl})$ & Nominal & $1=\mathrm{T} ; 0=\mathrm{F}$ \\
restecg & resting electrocardiographic & Nominal & $0,1,2$ \\
thalach & max heart rate & Numerical & \\
exang & exercise & Nominal & $1-\mathrm{Y}, 0-\mathrm{N}$ \\
oldpeak & depression induced by exercise with respect to rest & Numerical & \\
slope & Peak exercise & Nominal & $1,2,3$ \\
ca & \# of major vessels & Numerical & \\
thal & $\mathrm{t} 3$ - normal, t6 - fixed defect, $\mathrm{t} 7$ - reversable defect & Numerical & \\
Target & Diagnosis of disease & Numerical & Between 0,1 \\
\hline
\end{tabular}
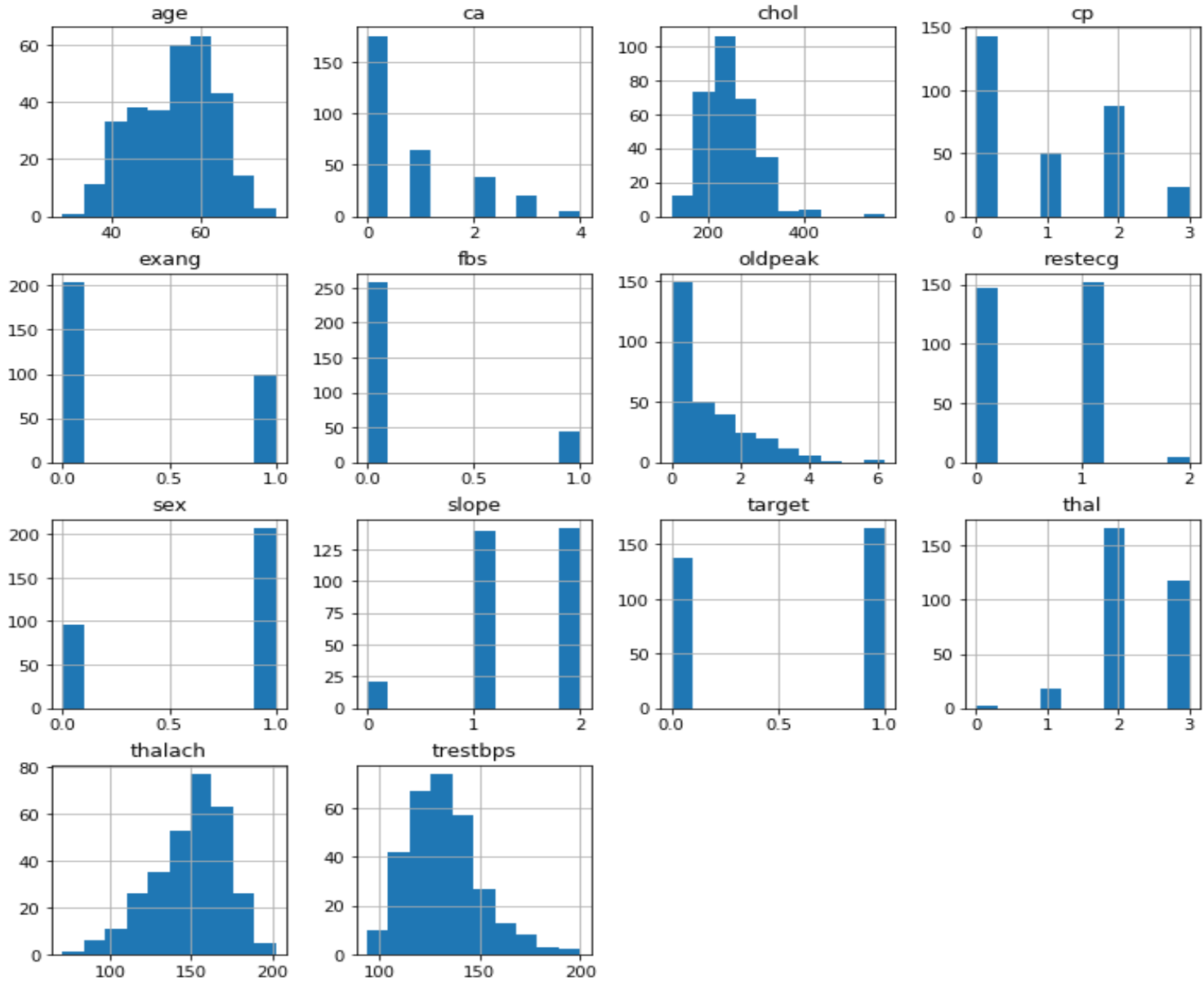

Figure 2. Histograms of data frame attributes 


\subsubsection{Proposed work}

The proposed convolutional architecture contains the input layer followed by a convolutional layer with 16 kernels along with activation function as ReLU, in the subsequent layer $25 \%$ of the nodes is dropped by dropout layer. Again, the convolutional layer was performed with eight kernels with previous parameters, also applied the dropout layer with $25 \%$. For prediction probability calculations, added an output layer.

The proposed architecture is shown in Figure 3. The cleaned data is split into $80 \%$ training and $20 \%$ testing for training and testing purposes. The same dataset is tested with different machine learning classifiers such as Logistic Regression (LR), $\mathrm{NB}, \mathrm{KNN}$, and SVM with different kernels, such as linear and $\mathrm{RBF}$ and simple neural networks. In this paper, we proposed a CNN to predict the accuracy of whether a patient had a cardio disease or not. 89.91 training accuracy and $86.83 \%$ testing accuracy.

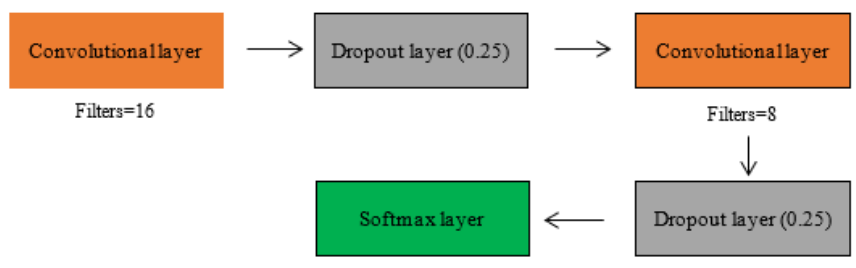

Figure 3. Proposed architecture

The proposed convolutional network model accuracy is compared with the existing ML models which were depicted in Table 2. Logistic regression achieved $89.91 \%$. Naïve Bayes achieved $80.62 \%$ training accuracy and $77.04 \%$ testing accuracy. KNN achieved $79.76 \%$ training accuracy and $68.86 \%$ testing accuracy. SVM (Linear) achieved $90.61 \%$ training accuracy and $86.83 \%$ testing accuracy. SVM (RBF) achieved $85.43 \%$ training accuracy and $81.96 \%$ testing accuracy. Neural Network achieved $88.95 \%$ training accuracy and $86.97 \%$ testing accuracy. The proposed network achieved $95.04 \%$ training accuracy and $94.78 \%$ testing accuracy. The graphical representation of existing and proposed method accuracies is shown in Figure 4. Figure 5 represents the ROC curves of LR, NB, KNN, SVM (Linear), SVM (RBF), and Neural Networks.

Table 2. Accuracies of different methods on Cleveland dataset

\begin{tabular}{ccc}
\hline Model & Training Accuracy & Testing Accuracy \\
\hline Logistic Regression & 89.91 & 86.83 \\
Naive Bayes & 80.62 & 77.04 \\
KNN & 79.76 & 68.86 \\
SVM Linear & 90.61 & 85.29 \\
Neural Network & 85.43 & 81.96 \\
Proposed Network & 88.95 & 86.97 \\
\hline
\end{tabular}

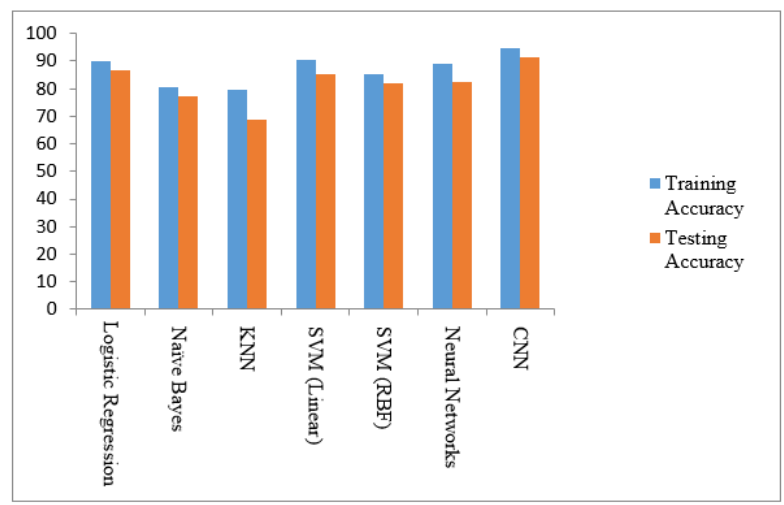

Figure 4. Graphical representation of accuracies in different models

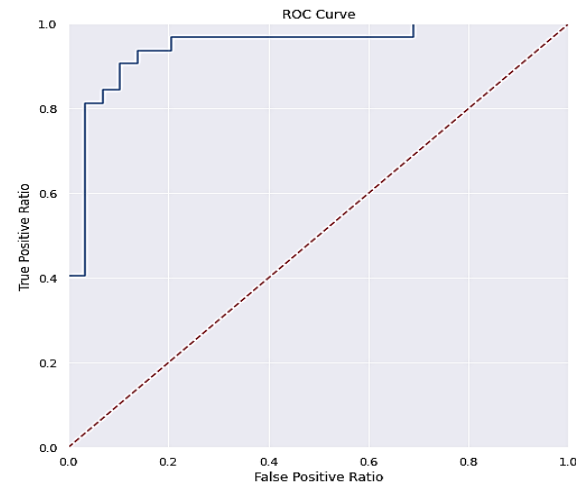

a. Logistic regression

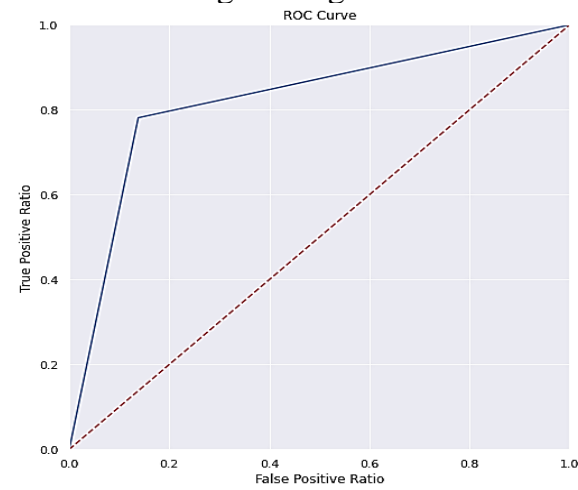

d. SVM (RBF)

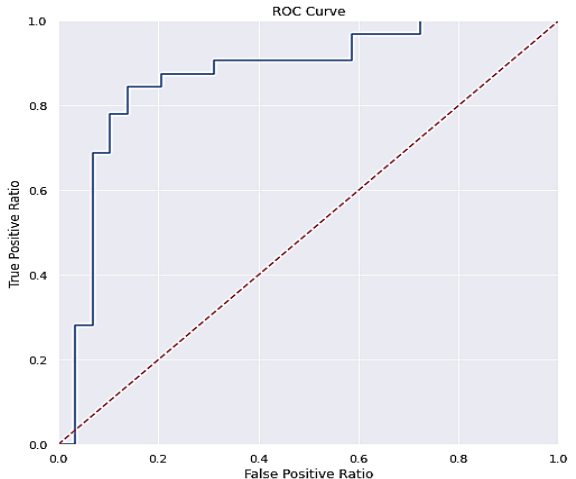

b. Naïve Bayes

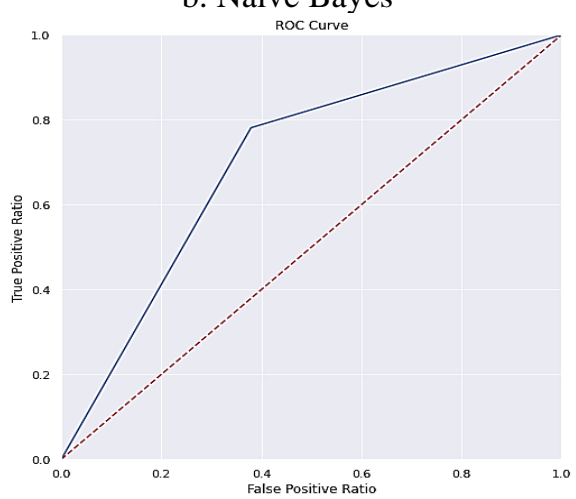

e. $\mathrm{KNN}$

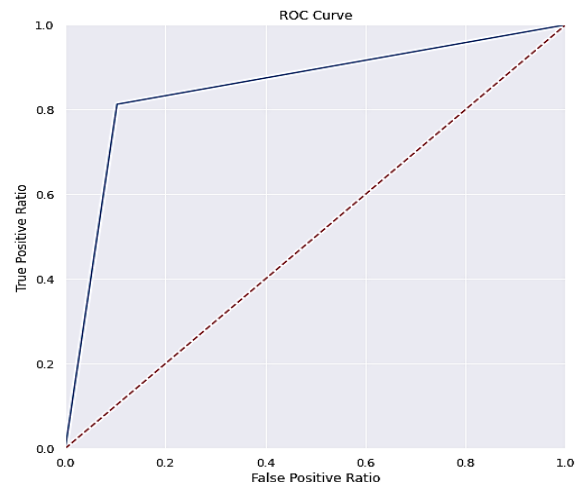

c. SVM (Linear)

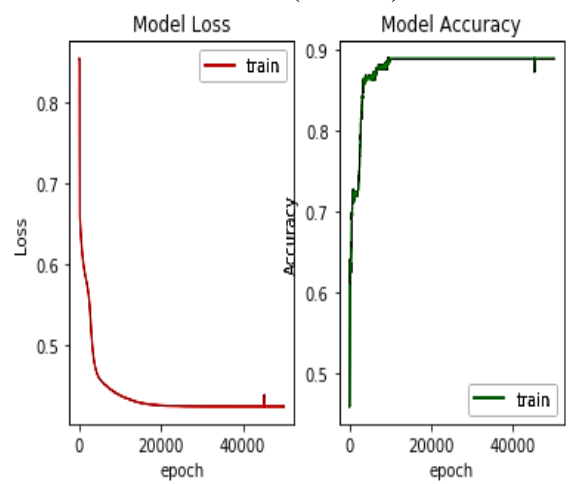

f. Neural networks

Figure 5. ROC curves 


\section{CONCLUSION}

Many of the state-of-the-art algorithms are not suitable to predict CVD disease correctly. Even doctors are also unable to predict the disease accurately. So, the proposed system supports medicos for prediction. In this script, we proposed a convolutional neural network-based model to predict the disease. And also, the paper gave a comparison between proposed work and state-of-the-art algorithms. The proposed model consists of two convolutional layers, two dropout layers, and an output layer. The reported accuracy by this model is $94.78 \%$ to predict disease on the UCI-ML Cleveland dataset. The proposed network deals with a large volume of data. Another advantage of this model is preprocessing, feature extraction and prediction done by the model itself, whereas the existing algorithm used different methods for each task.

\section{REFERENCES}

[1] Bhatnagar, P., Wickramasinghe, K., Wilkins, E., Townsend, N. (2016). Trends in the epidemiology of cardiovascular disease in the UK. Heart, 102(24): 19451952. https://doi.org/10.1136/heartjnl-2016-309573

[2] Jabbar, M.A., Chandra, P., Deekshatulu, B.L. (2012). Prediction of risk score for heart disease using associative classification and hybrid feature subset selection. 2012 12th International Conference on Intelligent Systems Design and Applications (ISDA), Kochi, pp. 628-634. https://doi.org/10.1109/ISDA.2012.6416610

[3] Beunza, J.J., Puertas, E., García-Ovejero, E., Villalba, G., Condes, E., Koleva, G., Hurtado, C., Landecho, M.F. (2019). Comparison of machine learning algorithms for clinical event prediction (risk of coronary heart disease). Journal of Biomedical Informatics, 97: 103257. https://doi.org/10.1016/j.jbi.2019.103257

[4] Zhao, L., Liu, C., Wei, S., Liu, C., Li, J. (2019). Enhancing detection accuracy for clinical heart failure utilizing pulse transit time variability and machine learning. IEEE Access, 7: 17716-17724. https://doi.org/10.1109/ACCESS.2019.2895230

[5] Chen, R., Lu, A., Wang, J., Ma, X., Zhao, L., Wu, W., Du, Z., Fei, H., Lin, Q., Yu, Z., Liu, H. (2019). Using machine learning to predict one-year cardiovascular events in patients with severe dilated cardiomyopathy. European Journal of Radiology, 117: 178-183. https://doi.org/10.1016/j.ejrad.2019.06.004

[6] Mezzatesta, S., Torino, C., De Meo, P., Fiumara, G., Vilasi, A. (2019). A machine learning-based approach for predicting the outbreak of cardiovascular diseases in patients on dialysis. Computer Methods and Programs in Biomedicine, 177: 9-15.

[7] Awan, S.M., Riaz, M.U., Khan, A.G. (2018). Prediction of heart disease using artificial neural network. VFAST Transactions on Software Engineering, 13(3): 102-112. http://dx.doi.org/10.21015/vtse.v13i3.511

[8] Gjoreski, M., Simjanoska, M., Gradišek, A., Peterlin, A., Gams, M., Poglajen, G. (2017). Chronic heart failure detection from heart sounds using a stack of machinelearning classifiers. 2017 International Conference on Intelligent Environments (IE), Seoul, pp. 14-19. https://doi.org/10.1109/IE.2017.19

[9] Alaa, A.M., Bolton, T., Di Angelantonio, E., Rudd, J.H., van Der Schaar, M. (2019). Cardiovascular disease risk prediction using automated machine learning: A prospective study of 423,604 UK Biobank participants. PloS One, 14(5): 0213653. https://doi.org/10.1371/journal.pone.0213653

[10] Abdar, M., Książek, W., Acharya, U.R., Tan, R.S., Makarenkov, V., Pławiak, P. (2019). A new machine learning technique for an accurate diagnosis of coronary artery disease. Computer Methods and Programs in Biomedicine, 179: 104992. https://doi.org/10.1016/j.cmpb.2019.104992

[11] Tang, X., Ma, Z., Hu, Q., Tang, W. (2019). A real-time arrhythmia heartbeats classification algorithm using parallel delta modulations and rotated linear-kernel support vector machines. IEEE Transactions on Biomedical Engineering, 67(4): 978-986. https://doi.org/10.1109/TBME.2019.2926104

[12] Squire, K.J., Zhao, Y., Tan, A., Sivashanmugan, K., Kraai, J.A., Rorrer, G.L., Wang, A.X. (2019). Photonic crystal-enhanced fluorescence imaging immunoassay for cardiovascular disease biomarker screening with machine learning analysis. Sensors and Actuators B: Chemical, 290: 118-124. https://doi.org/10.1016/j.snb.2019.03.102

[13] Kalluri, H.K., Prasad, M.V., Agarwal, A. (2012). Palmprint identification based on wide principal lines. Proceedings of the International Conference on Advances in Computing, Communications and Informatics, pp. 918-924. https://doi.org/10.1145/2345396.2345544

[14] Alizadehsani, R., Abdar, M., Roshanzamir, M., Khosravi, A., Kebria, P.M., Khozeimeh, F., Acharya, U.R. (2019). Machine learning-based coronary artery disease diagnosis: A comprehensive review. Computers in Biology and Medicine, 111: 103346. https://doi.org/10.1016/j.compbiomed.2019.103346

[15] Kumar, N.K., Vigneswari, D. (2019). Hepatitisinfectious disease prediction using classification algorithms. Research Journal of Pharmacy and Technology, 12(8): 3720-3725. https://doi.org/10.5958/0974-360X.2019.00636.X

[16] Sajja, T.K., Devarapalli, R.M., Kalluri, H.K. (2019). Lung cancer detection based on CT scan images by using deep transfer learning. Traitement du Signal, 36(4): 339344. https://doi.org/10.18280/ts.360406

[17] Balugani, E., Lolli, F., Butturi, M.A., Ishizaka, A., Sellitto, M.A. (2020). Logistic regression for criteria weight elicitation in PROMETHEE-based ranking methods. In: Ahram T., Karwowski W., Vergnano A., Leali F., Taiar R. (eds) Intelligent Human Systems Integration 2020. Advances in Intelligent Systems and Computing, vol 1131. Springer, Cham. https://doi.org/10.1007/978-3-030-39512-4_74

[18] Kaur, G., Oberoi, A. (2020). Novel approach for brain tumor detection based on Naïve Bayes classification. In: Sharma N., Chakrabarti A., Balas V. (eds) Data Management, Analytics and Innovation. Advances in Intelligent Systems and Computing, vol 1042. Springer, Singapore. https://doi.org/10.1007/978-981-32-99498_31

[19] Land, W.H., Schaffer, J.D. (2020). The support vector machine. In the Art and Science of Machine Intelligence, Springer, Cham, pp. 45-76. https://doi.org/10.1007/9783-030-18496-4_2 
[20] Tang, H., Xu, Y., Lin, A., Heidari, A.A., Wang, M., Chen, H., Luo, Y., Li, C. (2020). Predicting green consumption behaviors of students using efficient firefly grey wolfassisted K-nearest neighbor classifiers. IEEE Access, 8: 35546-35562.

https://doi.org/10.1109/ACCESS.2020.2973763
[21] Christopher, B. (2016). Replication Data for: Cleveland Heart Disease. https://doi.org/10.7910/DVN/QWXVNT, Harvard Dataverse, V1, UNF:6:uUXnE2XOKvaGcPfH8fzDpw== [fileUNF], accessed on 26 February 2020. 